\title{
The relationship between cadence, pedalling technique and gross efficiency in cycling
}

\author{
Stig Leirdal · Gertjan Ettema
}

Received: 20 October 2010 / Accepted: 7 March 2011 / Published online: 25 March 2011

(C) The Author(s) 2011. This article is published with open access at Springerlink.com

\begin{abstract}
Technique and energy saving are two variables often considered as important for performance in cycling and related to each other. Theoretically, excellent pedalling technique should give high gross efficiency (GE). The purpose of the present study was to examine the relationship between pedalling technique and GE. 10 well-trained cyclists were measured for GE, force effectiveness (FE) and dead centre size (DC) at a work rate corresponding to $\sim 75 \%$ of $\mathrm{VO}_{2}$ max during level and inclined cycling, seat adjusted forward and backward, at three different cadences around their own freely chosen cadence (FCC) on an ergometer. Within subjects, FE, DC and GE decreased as cadence increased $(p<0.001)$. A strong relationship between FE and GE was found, which was to great extent explained by FCC. The relationship between cadence and both $\mathrm{FE}$ and GE, within and between subjects, was very similar, irrespective of FCC. There was no difference between level and inclined cycling position. The seat adjustments did not affect FE, DC and GE or the relationship between them. Energy expenditure is strongly coupled to cadence, but force effectiveness, as a measure for pedalling technique, is not likely the cause of this relationship. FE, DC and GE are not affected by body orientation or seat adjustments, indicating that these parameters and the relationship between them are robust to coordinative challenges within a range of cadence, body orientation and seat position that is used in regular cycling.
\end{abstract}

Communicated by Jean-René Lacour.

S. Leirdal $(\bowtie) \cdot$ G. Ettema

Department of Human Movement Science,

Norwegian University of Science and Technology (NTNU),

Dragvoll Idrettssenter 3 etg, 7491 Trondheim, Norway

e-mail: stig.leirdal@svt.ntnu.no
Keywords Cadence $\cdot$ Inclined $\cdot$ Level $\cdot$ Pedalling · Technique

\section{Introduction}

In cycling, not only the work capacity but also a proper technical execution of the propulsive movements is often considered to be important for performance. In cycling, force effectiveness (FE) is often used as a parameter to indicate the quality of pedalling technique (e.g., Patterson and Pearson 1983; Ericson and Nisell 1988; Coyle et al. 1991; Sanderson 1991; Sanderson and Black 2003; Zameziati et al. 2006; Candotti et al. 2007; Korff et al. 2007). FE is the ratio between the force directed $90^{\circ}$ on the crank arm and the total resultant force on the pedal. Furthermore, it is generally believed that high gross efficiency (GE) is related to good technique in general and high FE specifically (e.g., Zameziati et al. 2006; Candotti et al. 2007). In a mechanically effective pedalling technique with high FE a large component of the generated force is directed perpendicularly on the crank arm. Forces directed otherwise, i.e., radially to the crank, do not contribute to work rate and the associated energy cost is wasted. Thus, in principle, FE affects $\mathrm{GE}$ in a direct manner. A number of studies have demonstrated a moderate to strong relationship between FE and GE (e.g., Zameziati et al. 2006; Candotti et al. 2007).

On the other hand, various studies have shown that for an effective and powerful crank cycle, one must generate considerable radial forces. This is due to the mechanical constraints within the rider-bicycle system (Kautz and Hull 1993). Because of the constraints, it is not a priori so that the most effective force is generated with the least muscular effort. Furthermore, inertial and gravitational forces add to the ineffective component of pedal forces 
(Kautz and Hull 1993), which are of no physiological consequence. This raises questions on both the origin as well as the significance of the apparent relationship between FE and GE.

GE indicates the total metabolic rate, including muscle work rate, for a given external work rate, and $\mathrm{FE}$ is the resultant outcome of all muscle activation. Thus, an FE-GE relationship that is unaffected by other factors would indicate that the total amount of muscle work done at a given external work rate (via GE) and the net coordinative outcome (via FE) are tightly coupled.

Cadence is shown to affect both FE (Patterson and Pearson 1983; Sanderson 1991; Candotti et al. 2007; Lorås et al. 2009) and GE (Seabury et al. 1977; Coast and Welch 1985; Belli and Hintzy 2002; Foss and Hallen 2004, 2005; Samozino et al. 2006; Hansen and Sjøgaard 2007). Body orientation (e.g., inclination as in inclined cycling) and seating position (e.g., differences between road cycling, time trial and triathlon cycling, see Faria et al. 2005) are factors that possibly affect both FE and/or GE (e.g., Bertucci et al. 2005; Brown et al. 1996; Caldwell et al. 1998, 1999; Millet et al. 2002; Faria et al. 2005; Heil et al. 1997; Price and Donne 1997; Ricard et al. 2006; Umberger et al. 1998; Welbergen and Clijsen 1990). While both FE and GE are studied quite extensively related to these factors, relatively little is done on the relationship between FE and GE, i.e., if the relationship is independent of other factors. Particularly, the role of cadence is of importance and may explain any relationship between $\mathrm{FE}$ and GE if it affects both in a similar fashion.

Recently, Leirdal and Ettema (2010) introduced a new pedalling technique parameter, which described the dead centre (DC) and was defined as the minimum power divided by the average power during the pedal stroke. It had a stronger relationship with GE than FE and it was, unlike FE, not affected by inertial forces that increase with cadence. Thus, it could be hypothesised that DC is not affected by cadence in the way that $\mathrm{FE}$ and GE are. The influence of cadence on DC has not been investigated before. By studying, in detail, how the relationship between on the one hand FE and DC (technique) and on the other GE (energy expenditure) is affected by cadence, more insight may be obtained in how cycling technique and energy expenditure are related.

In the present study, we therefore investigated the relationship between pedalling technique (FE and DC) and GE, over three cadences, for level and inclined cycling position, and for three seating positions. We took the freely chosen cadence (FCC) of the cyclists as a departure point to set the range of cadences. This increased the total range of cadences and allowed for studying the effect of absolute (inter-individual differences) as well as relative cadence (intra-individual differences).

\section{Method}

Subjects

The study was approved by the local ethics committee and all participants signed an informed written consent before participating in the study. Ten well-trained cyclists, at a national and regional level, participated in the study. The Table 1.

Protocol and analysis

All participants met in the lab on two occasions. On the first occasion, they performed an incremental test at freely chosen cadence (FCC) on a Velotron ergometer with a computer-controlled electro-magnetic brake mechanism (Velotron, Racermate inc., Washington). This ergometer generates a constant power condition, independent of cadence. The participants wore cycling shoes and the seat and handle bar position on the ergometer was adjusted to the preferred sitting position for each participant. During the test, the participants did not receive any information about their pedal rate. After a 10-min warm-up at $100 \mathrm{~W}$, the participants performed an increasing work rate protocol that started at $100 \mathrm{~W}$ and had a $50 \mathrm{~W}$ increment every 2 min until exhaustion. Exhaustion was defined as meeting three of the four following criteria: (1) within 5 BPM from the participants self-reported maximal heart rate (HRmax), (2) above $7.5 \mathrm{mmol}^{-1}$ in blood lactate concentration, (3) the respiratory exchange ratio $(\mathrm{RER})>1.1$, and (4) a $\mathrm{VO}_{2}$ which stops increasing or starts decreasing with increased work rate. Pedal rate, oxygen consumption, and heart rate were measured continuously.

Gas exchange values were measured by open-circuit indirect calorimetry using an Oxycon Pro apparatus (Jaeger $\mathrm{GmbH}$, Hoechberg, Germany). Before each measurement, the $V \mathrm{O}_{2}$ and $V \mathrm{CO}_{2}$ gas analyzers were calibrated using high-precision gases $\left(16.00 \pm 0.04 \% \mathrm{O}_{2}\right.$ and $5.00 \pm 0.1 \%$ $\mathrm{CO}_{2}$, Riessner-Gase $\mathrm{GmbH} \& \mathrm{co}$, Lichtenfels, Germany). The flow meter was calibrated with a 3-L volume syringe (Hans Rudolph Inc., Kansas City, MO). Heart rate (HR) was measured with a heart rate monitor (Polar S610, Polar Electro OY, Kempele, Finland), using a 5-s interval for data storage. $V \mathrm{O}_{2} \max$ was defined as the highest 1-min

Table 1 Physical characteristics of the participants in study

\begin{tabular}{llrlll}
\hline & $\begin{array}{l}\text { Age } \\
\text { (years) }\end{array}$ & $\begin{array}{l}\text { Height } \\
(\mathrm{cm})\end{array}$ & $\begin{array}{l}\text { Body } \\
\text { mass }(\mathrm{kg})\end{array}$ & $\begin{array}{l}\mathrm{VO}_{2 \text { peak }} \\
\left(\mathrm{ml} \mathrm{kg} \mathrm{min}^{-1}\right)\end{array}$ & $\begin{array}{l}\text { Maximal } \\
\text { aerobic } \\
\text { power (watt) }\end{array}$ \\
\hline Avg & 23.4 & 183.2 & 77.3 & 58.1 & 370 \\
Std & 11.7 & 6.3 & 9.5 & 3.3 & 42 \\
\hline
\end{tabular}



study. a Normal condition. b Inclined condition. c Seat preferred backward and forward position
Fig. 1 Conditions in present
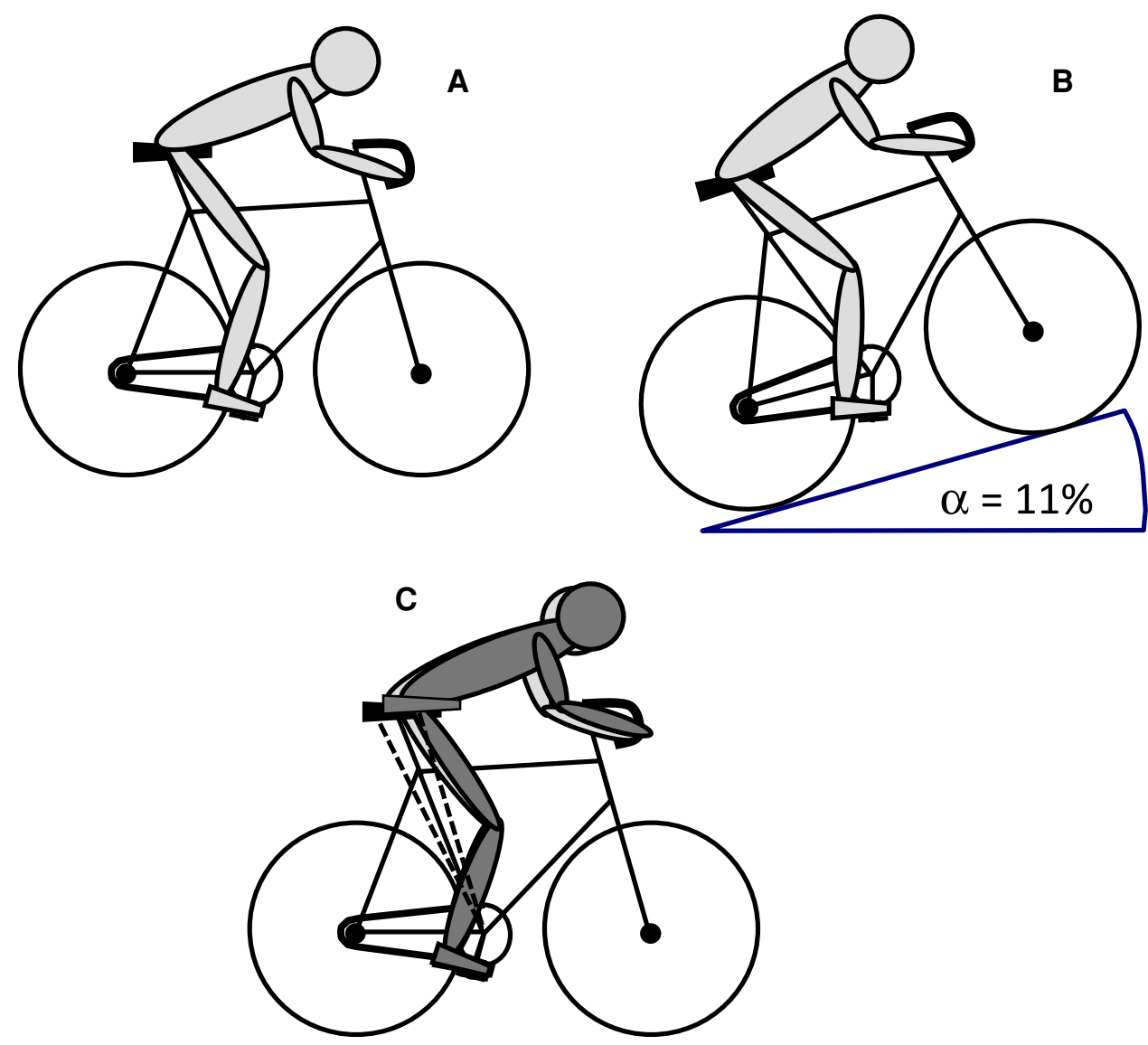

average $V_{2}$ during the test. Maximal heart rate was defined as the highest value that was attained, in average over a 5 -s period at the final stage of the protocol. Blood lactate concentration was measured $2 \mathrm{~min}$ after completion of the $V \mathrm{O}_{2}$ max test $\left(10.6 \mathrm{mmol}^{-1} \pm 1.9\right)$ by taking $5 \mu \mathrm{L}$ samples from the fingertip by a Lactate Pro LT-1710t (ArkRay Inc, Kyoto, Japan). This system was validated in literature (Medbø et al. 2000; Pyne et al. 2000; Baldari et al. 2009).

The second occasion, 3 days after the incremental test, all participants performed a protocol consisting of eight repetitions of 5 min cycling at a work rate that was estimated to elicit $\sim 80 \%$ of their $V_{2}$ max as was determined in the first test. Also during this test, the participants wore cycling shoes. The efforts were done at level and in tilted (11\%, i.e., $6.3^{\circ}$ inclined) position (Fig. $1 \mathrm{~b}$ ), all at preferred seat position. In the tilted position, the entire ergometer was tilted by elevating the front. Both positions (level and tilted) were performed at three cadences (FCC, FCC -10 and FCC $+10 \mathrm{rpm}$ ), giving six conditions. In addition, the level position was also performed with the seat moved forward and backward from the preferred position, giving two additional conditions. Corresponding seat adjustments were made in height as well, such that the angle of the line between crank centre and (rock point of the) seat was rotated by approximately $3^{\circ}$ in both directions. This led to a similar total angle change of about $6^{\circ}$ as in levels versus inclined position. For all forward and backward positions the distance between hip (major trochanter) and crank centre was unaltered in comparison with preferred seat position (Fig. 1c). This was done using a tape measure. The handlebars were moved in the same manner. Thus, in these conditions, the orientation of the upper body was not altered.

During all tests on the second occasion, the participants received continuous feedback about their cadence and were asked to keep it at a preset level. The FCC was set as the average cadence that was used the last minute during the incremental test at the work rate increment nearest $80 \%$ of $\mathrm{VO}_{2}$ max. To avoid any effect of fatigue, learning effect, or drift of energy expenditure in the statistical analysis, all conditions were done in a different order for each participant. Oxygen consumption and heart rate were measured continuously. GE was calculated as the ratio of work rate over metabolic cost rate as calculated from $V \mathrm{O}_{2}$ and RER. All measurements on $\sim 80 \% V \mathrm{O}_{2}$ max showed RER values below 1.0 (RER was $0.89 \pm 0.03$ ) indicating no significant anaerobic contribution. Kinetics was sampled for 5 times for $10 \mathrm{~s}$ at the end of each minute during the 5-min work periods.

Crank and pedal kinematics were recorded using a ProReflex (Qualisys, Sweden) 3D motion capture system with 
8 cameras in the same way as described by Ettema et al. (2009). Two spherical reflective markers were placed on extensions of both pedals in the sagittal plane of cyclist and bicycle. The positions of these markers were used to determine pedal orientation and crank angle. Both pedals were equipped with two force cells (Model 9363, Revere, capacity $250 \mathrm{~kg}$ per cell, The Netherlands), detecting pedal normal and shear forces (Ettema et al. 2009). The pedals were calibrated by applying full normal forces and full shear forces of known magnitude. A constant proportional crosstalk between the normal and shear forces of a single pedal was detected $(<3 \%)$ and taken into account by building a gain matrix.

All data were recorded using the QTM software (Qualisys, Sweden) at a sample rate of $500 \mathrm{~Hz}$ and further processed in Matlab (Mathworks, US). All data were lowpass filtered $(10 \mathrm{~Hz}, 8$ th order, zero lag Butterworth). After correction for acceleration artefacts (Ettema and Huijing 1994), pedal normal and shear forces were transformed to crank shear and normal forces by rotation of the coordination system from pedal to crank using the angle between pedal and crank as calculated from the kinematical data. The vector sum of right and left pedal forces (in the crank coordinate system) was used for further analysis (Lorås et al. 2009). This leads to higher FE values than considering the pedals separately, mainly because of the elimination of the negative effect of gravity during the up-stroke.

Normal crank force was considered to be the effective force component. Thus, the ratio of normal force over total force was defined as FE. FE was calculated as average of the $5 \times 10 \mathrm{~s}$ measurements from each 5 -min work period.

DC was defined as the lowest work rate (average of top and bottom dead centre) divided by the average work rate (Leirdal and Ettema 2010). Thus, this is a parameter describing the evenness of work rate generation; $100 \%$ indicates a perfect circular work rate generation, whereas $0 \%$ indicates that the work rate at the DC equals zero.

Power was calculated as the product of crank moment (i.e., effective (normal) crank force $\times$ crank arm) and crank angular velocity. Continuous crank angular velocity was calculated from crank angles using a 5-point differentiating filter. The average crank cycle (for all variables) was calculated by interpolation of the crank angle-variable data to 360 samples, i.e., 1 sample per degree crank angle (Ettema et al. 2009).

To investigate how technique (FE, DC) and efficiency of energy consumption (GE) relate to each other, and how cadence may affect this relationship, we performed correlation matrix analysis as well as multiple regression analysis for GE with FE, DC, FCC and work rate as independent variables.

\section{Statistics}

All statistics were performed using Statistical Package for Social Sciences 15.0 (SPSS). The analysis consisted of two parts. Firstly, to confirm or refute findings in the literature, the general effect of cadence (in the range of $20 \mathrm{rpm}$ around FCC) and position on the variables of interest was examined: the intra-individual effects of position and cadence on GE, FE, and DC was analysed using a 2-way ANOVA (cadence and body position) and a 1-way ANOVA (seat position). FCC and absolute work rate were implemented as covariates. The second and main part of the analysis regarded the effect of cadence on the relationship between technique and energy expenditure: the inter-individual relationships between GE (dependent) and FE, DC, FCC, and work rate (independent variables) was performed by multiple regression analysis at the three cadences. Furthermore, Pearson's correlations between variables were compared. This approach could not only indicate if, but also how FE and GE are related. Normality of the data distribution was checked with the one-sample Kolmogorov-Smirnov test. All data were considered normally distributed (all $p$ values $>0.337$ ). The significance level was set at $p<0.05$.

\section{Results}

The FCC in the main experiment (at the predicted work rate of $80 \% \mathrm{VO}_{2} \max$, averaging $210 \mathrm{~W}$ ) was $96 \pm 9.1 \mathrm{rpm}$ (range 75-107). Thus, the FCC -10 and FCC +10 conditions were performed on $86 \pm 9.1$ and $106 \pm 9.1 \mathrm{rpm}$, respectively. The load in this test elicited $75 \% \quad V_{2} \max$ instead of the predicted $80 \%$ during the steady-state cycling.

The 2-way ANOVA showed the following results for GE, FE and DC (average results are presented in Fig. 2). GE, FE and DC declined significantly $(p<0.001)$ with each increase in cadence in a similar way. Body orientation did not seem to have any effect on either FE ( $p=0.307), \mathrm{GE}(p=0.823)$ or DC $(p=0.166)$. Seat position had no effect on GE $(p=0.58)$ or DC $(p=0.978)$. The effect on FE was just not significant $(p=0.058)$. No significant cadence-orientation interaction was detected (FE, $p=0.090$; GE, $p=0.794$; and DC, $p=0.382$ ). The weak interaction trend for FE was localized between FCC and FCC +10 , which was different between the level and inclined orientation $(p=0.036)$. Both work rate (mean $210 \mathrm{~W}$, $\pm 40 \mathrm{~W}$ ) and FCC differed between participants in present study. Absolute work rate affects GE (Leirdal and Ettema 2009) and possibly FE and DC directly as well as being dependent on cadence. Therefore, we also treated absolute work rate and FCC as a covariate and examined its effect. The statistical findings using work rate as a covariate remained unaltered except for DC: cadence on FE, $p=0.001$; on DC, $p=0.164$; 

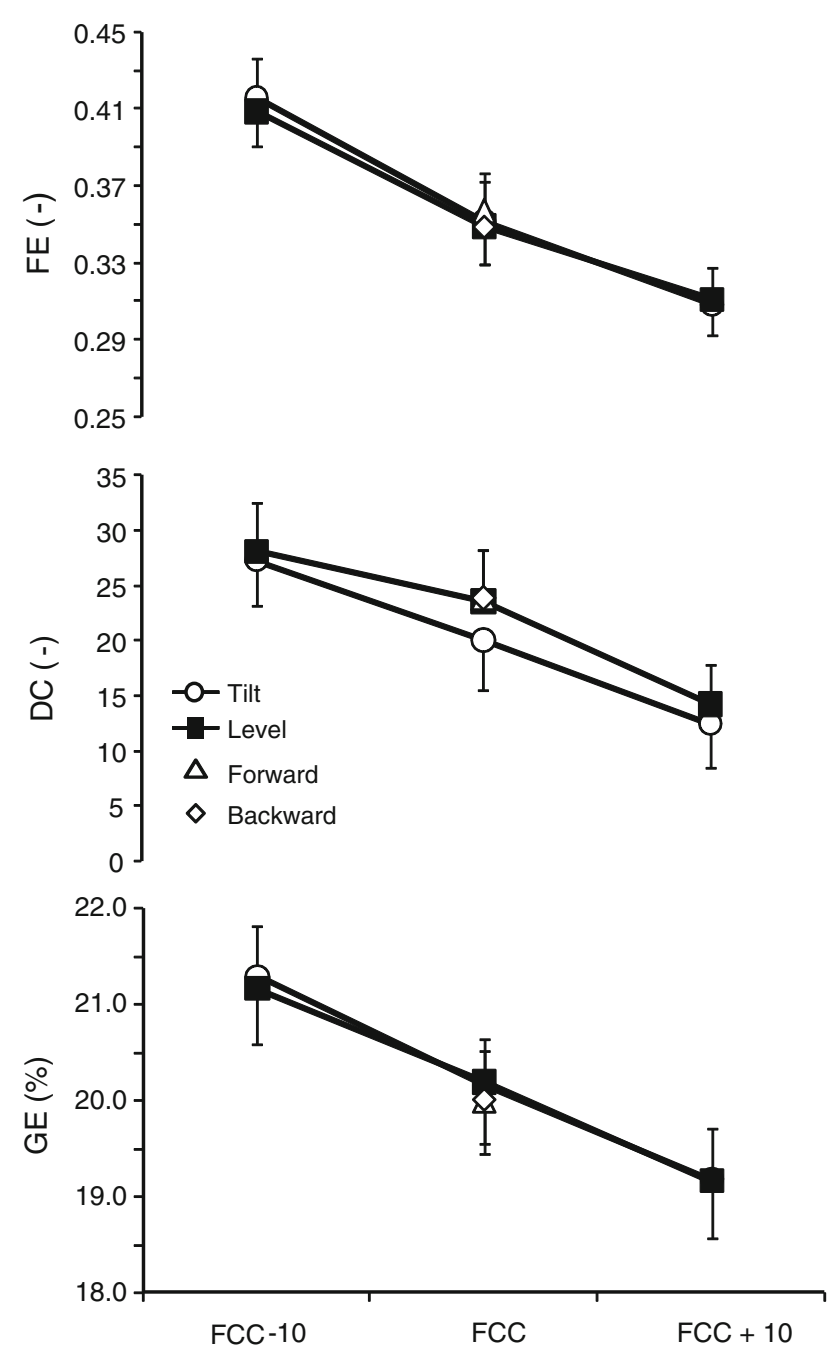

Fig. 2 The effect of cadence and body orientation on FE (top), DC (middle) and GE (bottom). FE, DC, and GE declined with increasing cadence $(p<0.001)$. Body orientation or seat adjustments did not have any impact. Vertical bars indicate SEM

on GE, $p=0.027$; orientation on $\mathrm{FE}, p=0.308$; on $\mathrm{DC}$, $p=0.553$; on GE, $p=0.172$; interaction on FE, $p=0.981$; on DC, $p=0.424$; on GE, $p=0.708$. Thus, the weak interaction trend on FE is explained by work rate differences. When using FCC as a covariate, all significant effects on FE, DC and GE disappeared (all $p>0.175$ ). In summary, within a subject, cadence was the main variable influencing FE, DC and GE in a similar way. Yet, these effects seemed to be related to the subject's FCC such that cyclists with a high FCC tend to show a cadence effect and those with low FCC did not.

Body orientation and seating position did not seem to have any effect on any of FE, DC, and GE. We double checked this by comparing the regression lines for the various conditions with the line of identity. In all cases, the regression estimate for FE, DC, and GE between any of the comparable conditions (4 at FCC, 2 at each other cadence) did not significantly differ from the line of identity (i.e., the intercept $=0$ and slope $=1$ ).
Thus, we could reduce the data by comparing the mean data for all conditions at each cadence. Table 2 shows a correlation matrix of all variables of interest. It appears that over the three cadences, inter-individual differences in FE and GE, and to a lesser extent also DC, are very consistent. FCC is strongly correlated with $\mathrm{FE}$ at all cadences, but not with DC. DC and FE are not related. Work rate correlates well with FCC, FE and GE. In the multiple regression analysis, for all three cadences, FCC was the only significant variable that remained, independent of variable selection method, significantly explaining the variance in GE. Still, in isolation, also FE and work rate showed significant correlations with GE (Table 2). In other words, FCC correlated strongest with GE (see Table 2), and $\mathrm{FE}$ and work rate did not significantly improve the prediction of GE, likely because they overlap in explaining the variance in FCC. Figure 3 shows FE and GE for all subjects and cadence against absolute cadence and indicates that FE and GE are tightly coupled to absolute cadence, irrespective of FCC. It is important to note that the intra-individual relationships (three points per subject, not shown in figure, but slopes are presented in the caption) were very similar to the overall relationship shown in the figure.

Although the mean values of both GE and FE are clearly affected by cadence (see ANOVA results above and Fig. 3), the changes are very consistent as indicated by the FE-FE and GE-GE correlations between cadences and the intra-class correlation (ICC) values (Table 2). Therefore, we also used the grand mean data of all conditions to estimate the average relationship between GE, FE and FCC. This led to a correlation between FE-GE of 0.660 , which was just significant $(p=0.037)$, between FCC and GE of $-0.812(p=0.004)$, and between FCC and FE of $-0.914(p<0.001)$.

\section{Discussion}

The present study showed that cadence has a strong negative and similar effect on both FE and DC, as well as GE, which is in line with the literature for both FE (Patterson and Pearson 1983; Sanderson 1991; Candotti et al. 2007; Lorås et al. 2009) and GE (Seabury et al. 1977; Coast and Welch 1985; Belli and Hintzy 2002; Foss and Hallen 2004, 2005; Samozino et al. 2006; Hansen and Sjøgaard 2007). The same effect for DC has, to our knowledge, not been reported before. The multiple regression analysis (plus correlation matrix) showed that both FE and GE are strongly affected by absolute cadence and thus by FCC. This finding is important when interpreting the relationship between FE and GE, which is probably not a causal one.

Within the range of frequencies used by this group of cyclists, there is clear and linear (negative) relationship between absolute cadence and GE, and even more so between cadence and FE (Fig. 3). It may be tempting to 
Table 2 Correlation matrix of significant relationships between FCC, work rate, and DE, FE, and GE at three cadences

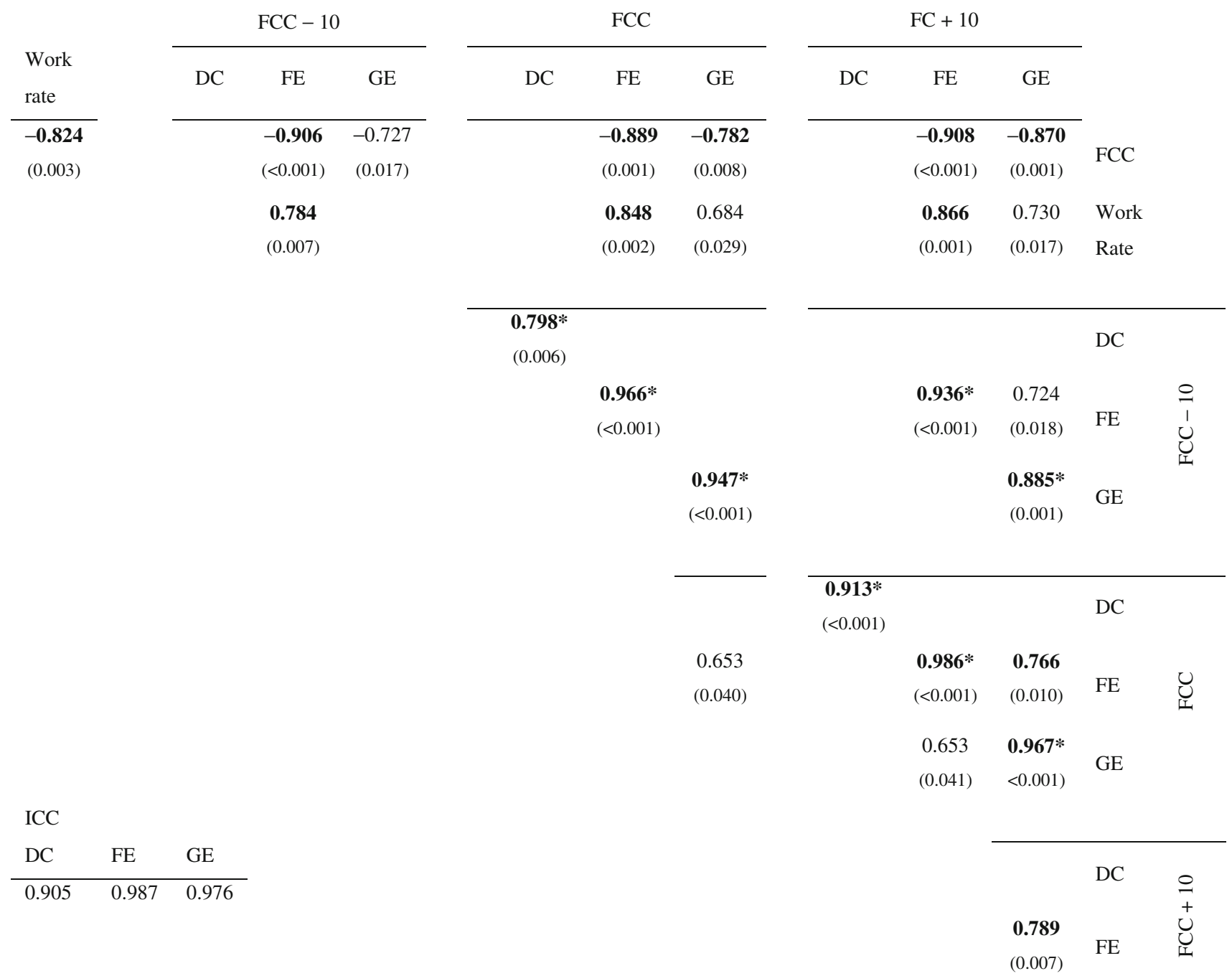

Correlations with a significance $<0.001$ are shown in bold. $p$ values are given in parentheses. These are generally very high, except for DC between FCC -10 and FCC +10 . The (average measure) ICC for DC, FE and GE are shown in the left bottom corner

* The correlations between the same variables but different cadence

conclude that the reduced FE with increasing cadence causes the cadence-GE relationship, in other words, that GE is directly affected by FE. However, this is unlikely because the cadence-induced FE reduction is explained by inertial mechanisms (Lorås et al. 2009) that have no bearing on energy consumption. The increased energy cost for moving the lower extremities is a more likely explanation. The fluctuations in internal kinetic energy (rotation of the lower extremities) increase with cadence. Although this energy flow can be utilised as external work (see Kautz and Neptune 2002; Ettema and Lorås 2009), it is likely associated with an increased energy cost and thus affects efficiency negatively. There are no studies, however, that have properly investigated the amount of this cost. (Note: These fluctuations are often referred to as internal work and considered fully as energy loss; various biomechanical analyses have shown this to be a flaw; for discussion, see e.g., Kautz and Neptune 2002 and Ettema and Lorås 2009.) A higher cadence will also increase the inertial, non-muscular component of the pedal forces (Kautz and Hull 1993; Ettema et al. 2009; Lorås et al. 2009), which are closely related to the kinetic energy fluctuations. An increase in inertial forces increases the radial force component in particular, and thereby affects FE in a negative way (e.g., Kautz and Hull 1993; Kautz and Neptune 2002; Lorås et al. 2009). Kautz and Neptune (2002) even argue that "effective force" is a misnomer. Thus, the similarity in cadence effect on efficiency and FE may be explained by two separate aspects of a common mechanism. However, this does not mean that force effectiveness is affecting efficiency. The inertial forces that affect the non-propulsive force component have, by definition, no associated metabolic cost. 

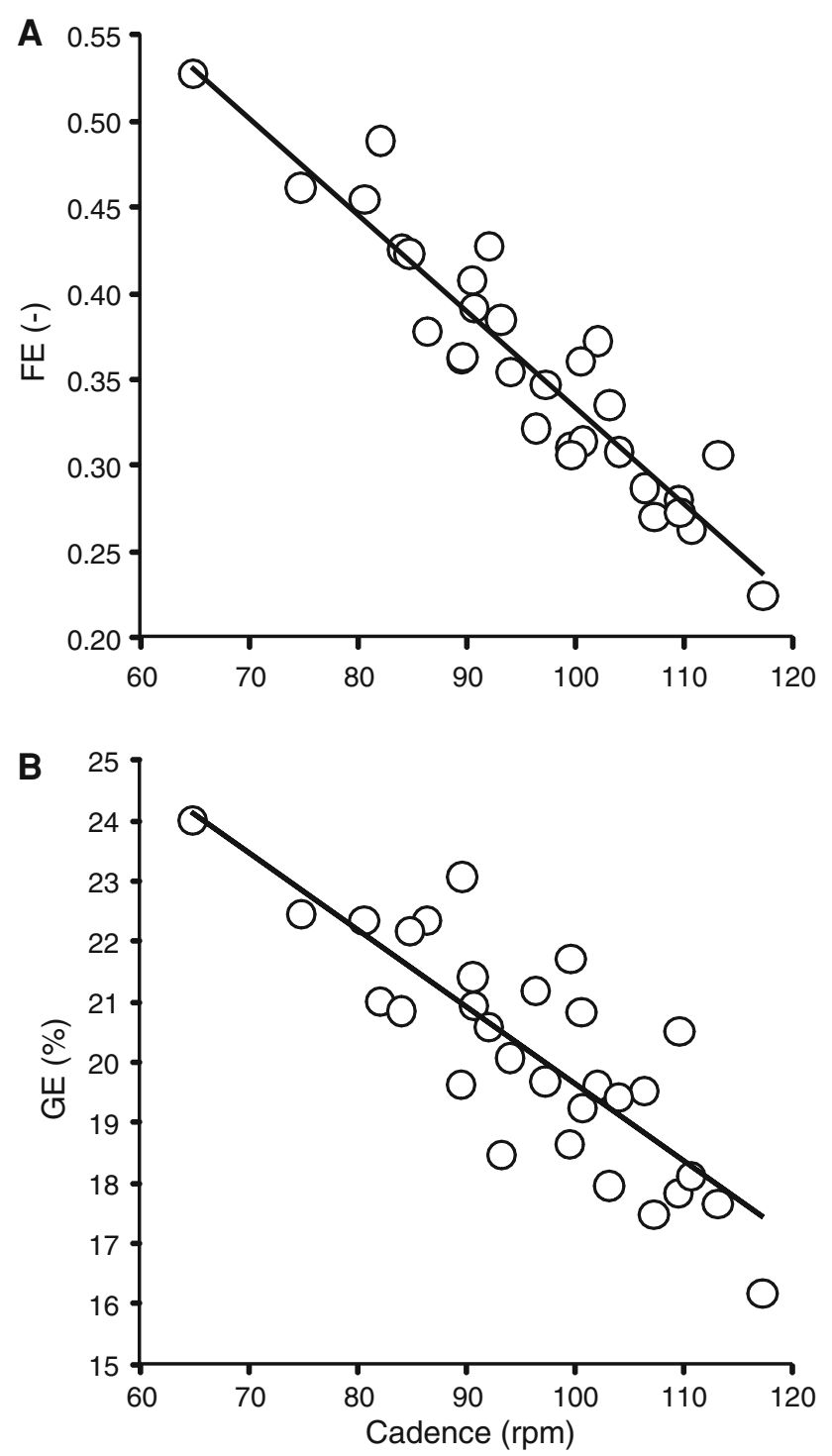

Fig. 3 FE (a) and GE (b) plotted against absolute cadence. Data are all subjects for all three cadences each subject performed at. The overall linear regression lines are indicated in the diagrams. The correlations were $-0.935(\mathrm{FE})$ and $-0.825(\mathrm{GE}), p<0.001$. The regression for each subject ( 3 data points) are not shown, but the slopes of these were not different from the slope of the regression of all data; FE: individual slopes $-0.0051 \pm 0.0009$ versus all data -0.0056 ; GE: individual slopes $-0.103 \pm 0.042$ versus all data -0.128

Lorås et al. (2009) showed that FE of the muscular force component is almost independent of cadence and relatively high (>0.8) (Lorås et al. 2009). Thus, the changes in effective crank forces are likely mainly caused by the inertial force component, which was also indicated by Kautz and Hull (1993) and Kautz and Neptune (2002). The present results confirm this notion that the cadence-FE relationship is caused by a mechanism that is extremely consistent, within and between subjects. Not only do all cyclists show the same trend when changing form FCC -10 to FCC + $10 \mathrm{rpm}$, but this trend is identical with the inter-individual difference that is created by choice of cadence (FCC) (Fig. 3a). Furthermore, the multiple regression analysis demonstrates that cadence (or FCC) rather than the associated FE determines GE. The increase of metabolic cost (decrease in efficiency) can therefore not be linked to the decrease in $\mathrm{FE}$, at least not in a direct causal manner. Beside the assumed metabolic cost of rotating the legs, extra costs may occur because high cadence requires additional muscle activity for coordination. Zameziati et al. (2006) reported a significant FE-GE relationship, determined over a range of work rates at $80 \mathrm{rpm}$ cadence. This may be explained in a similar way. When increasing work rate at one cadence, the ineffective inertial forces will remain constant while the propulsive force must increase to increase power. This will automatically lead to an enhanced FE which is not necessarily indicating an improved technique (work rate has a diminishing effect on the inertial force contribution). Work rate also has a positive effect on efficiency but via a different mechanism (see Ettema and Lorås 2009).

Absolute work rate co-varies negatively with FCC and may be a partial explanation for the cadence-GE relationship. Because of the general work rate-efficiency relationship (Ettema and Lorås 2009), a higher work rate (i.e., lower FCC) will result in a higher GE. However, the intraindividual effect of cadence on GE is not affected by the work rate that was applied. Thus, the work rate effect does not explain the entire relationship between cadence and GE.

Leirdal and Ettema (2010) found that inertial effects not to affect DC. Still, within each subject, cadence negatively affects DC when using a high FCC. Furthermore, Leirdal and Ettema (2010) reported an inter-individual relationship between DC and GE. Thus, it seems reasonable to suggest that the diminishing DC with increasing cadence explains the relationship between cadence and GE. However, the finding by Leirdal and Ettema (2010), i.e., the DC-GE relationship, was not reproduced in the present study, which leaves this proposed explanation open for debate. A reason for the contradicting results of this study and Leirdal and Ettema (2010) may be the type of bicycle-ergometer system that was used. Leirdal and Ettema (2010) used a racer bicycle with regular gears on resisting rollers, whereas in the present study a computer-controlled electromagnetic brake system was used. Leirdal and Ettema (2009) showed that these systems have a different outcome on the choice of cadence in relation to work rate. Thus, cycling technique (e.g., DC) may also have been affected by the choice of ergometer system. This may also explain the relative low FE values as compared with other studies (e.g., Dorel et al. 2009; Lorås et al. 2009; Sanderson and Black 2003; Hug et al. 2008). Our lower FE values cannot be explained by the method of calculation; Lorås et al. (2009) showed that this method leads to higher values rather than 
lower. The data by Lorås et al. (2009) were collected in the same laboratory with identical measurement equipment and calculation algorithms and a similar subject group. The only difference was the type of ergometer/bicycle that was used. This supports the notion that the type of ergometer may affect these technique values considerably.

There was no difference on any parameter between level and tilted cycling or between preferred, forward, and backward seat position. FE, DC and GE showed almost identical values and effects of cadence in both level and tilted cycling and for the three seat positions. This is quite a noteworthy finding as it suggests that the individual cyclist has his own pedalling characteristic that is unaffected by (upper) body orientation. The present results are in disagreement with the notion that cycling technique and thereby power production and energy consumption is affected by relatively small changes in body orientation as occur in practice (e.g., Caldwell et al. 1998; Heil et al. 1997; Price and Donne 1997). The high ICC values for FE, DC and GE between conditions confirm the notion that FE, DC and GE are very subject specific. The changes between the various orientation and seating conditions (about $6^{\circ}$ rotation) may appear marginal. This could explain the lack of any effect of these parameters. However, from a practical standing, these changes are quite large: to obtain a change of $3^{\circ}$ in the seatcrank angle, the seat was shifted approximately $4 \mathrm{~cm}$. Furthermore, changes in other technique variables caused by such position changes have been detected: unpublished results from our laboratory indicate that the $6^{\circ}$ rotation of the cyclist (inclined position) or the lower extremities (by seat position) cause a phase shift of the crank cycle (see also McGhie and Ettema 2011) of the same amount (i.e., 6 degrees); Umberger et al. (1998) reported relatively small but significant changes in power at maximal effort (about $4 \mathrm{~W}$ per degree seat-crank angle) and hip angles (about 1 degree degree $^{-1}$ ). Thus, the relatively small range of body orientation used in this study should not be considered as a limiting factor for detection of its effect on technique and energy consumption.

There are some limitations in the present study. The pedalling rates investigated $(86-106 \mathrm{rpm})$ are a relatively small range around the FCC for competitive cyclists that covers most cadences used in mass starts competitive cycling. However, the findings of this study cannot be generalised to a wider range of cadences that is used regularly in experimental studies and in other cycling disciplines. Furthermore, the ergometer used in present study may have influenced the choice of cadence (Leirdal and Ettema 2009) and might also affect pedalling dynamics.

In conclusion, energy expenditure is strongly coupled to cadence, but force effectiveness, as a measure for pedalling technique, is not likely the cause of this relationship. Along with other studies (Kautz and Hull 1993; Ettema et al.
2009; Lorås et al. 2009), we are inclined to conclude that FE is mostly affected by inertial forces, and thus the value of this parameter as a measure for technique should be questioned. Contrary to Leirdal and Ettema (2010), we do not find a significant relationship between DC and GE. Thus, the present study provides no indication for the notion that technique affects energy consumption. There was no significant effect of body orientation or seat position on GE, FE or DC, or on the relationship between them, indicating that these parameters and the relationship between them are robust to coordinative challenges within a range of cadence, body orientation and seat position that is used in regular cycling.

Open Access This article is distributed under the terms of the Creative Commons Attribution Noncommercial License which permits any noncommercial use, distribution, and reproduction in any medium, provided the original author(s) and source are credited.

\section{References}

Baldari C, Bonavolonta V, Emerenziani GP, Gallotta MC, Silva AJ, Gudetti L (2009) Accuracy, reliability, linearity of accutrend and Lactate Pro versus EBIO plus analyzer. Eur J Appl Physiol 107:105-111

Belli A, Hintzy F (2002) Influence of pedalling rate on the energy cost of cycling in humans. Eur J Appl Physiol 88:158-162

Bertucci W, Grappe F, Girard A, Betik A, Rouillon JD (2005) Effects of the crank torque profile when changing pedalling cadence in level ground and inclined road cycling. J Biomech 38:1003-1010

Brown DA, Kautz SA, Dairaghi CA (1996) Muscle activity patterns altered during pedalling at different body orientations. J Biomech 29(10):1349-1356

Caldwell GE, Li L, McCole SD, Hagberg JM (1998) Pedal and crank kinetics in inclined cycling. J Appl Biomech 14:245-259

Caldwell GE, Hagberg JM, McCole SD, Li L (1999) Lower extremity joint moments during inclined cycling. J Appl Biomech 15:166-181

Candotti CT, Ribeiro J, Soares DP, DeOliveira AR, Loss JF, Guimaraes ACS (2007) Effective force and economy of triathletes and cyclists. Sport Biomech 6(1):31-43

Coast JR, Welch HG (1985) Linear increase in optimal pedal rate with increased power output in cycle ergometry. Eur J Appl Physiol 53:339-342

Coyle EF, Feltner ME, Kautz SA, Hamilto MT, Montain SJ, Baylor AM, Abraham LD, Petrek GW (1991) Physiological and biomechanical factors associated with elite endurance cycling performance. Med Sci Sports Exerc 23(1):93-107

Dorel S, Drouet JM, Couturier A, Champoux Y, Hug F (2009) Changes of pedalling technique and muscle coordination during an exhaustive exercise. Med Sci Sports Exerc 41(6):1277-1286

Ericson MO, Nisell R (1988) Efficiency of pedal forces during ergometer cycling. Int J Sports Med 9:118-122

Ettema G, Huijing PA (1994) Frequency response of rat gastrocnemius medialis in small amplitude vibrations. J Biomech 27:1015-1022

Ettema G, Lorås H (2009) Efficiency in cycling: a review. Eur J Appl Physiol 106(1):1-14

Ettema G, Lorås H, Leirdal S (2009) The effects of cycling cadence on the phases of joint power, crank power, force and FE. J Electromyogr Kinesiol 19(2):94-101

Faria EW, Parkel DL, Faria IE (2005) The science of cycling: factors affecting performance-Part 1. Sport Med 35:285-312 
Foss Ø, Hallen J (2004) The most economical cadence increases with increasing workload. Eur J Appl Physiol 92:443-451

Foss $\varnothing$, Hallen J (2005) Cadence and performance in elite cyclists. Eur J Appl Physiol 93:453-462

Hansen EA, Sjøgaard G (2007) Relationship between efficiency and pedal rate in cycling: significance of internal power and muscle fiber composition. Scand J Med Sci Sports 17:408-414

Heil DP, Derrick TR, Whittlesey S (1997) The relationship between preffered and optimal positioning during submaximal cycle ergometry. Eur J Appl Physiol 75:160-165

Hug F, Drouet JM, Champoux Y, Couturier A, Dorel S (2008) Interindividual variability of electromyographic patterns and pedal force profiles in trained cyclists. Eur J Appl Physiol 104(4):667-678

Kautz SA, Hull ML (1993) A theoretical basis for interpreting the force applied to the pedal in cycling. J Biomech 26(2):155-165

Kautz SA, Neptune RR (2002) Biomechanical determinants of pedalling energetics: internal and external work are not independent. Exerc Sport Sci Rev 30(4):159-165

Korff T, Romer LM, Mayhew I, Martin JC (2007) Effect of pedalling technique on mechanical effectiveness and efficiency in cyclists. Med Sci Sports Exerc 39(6):991-995

Leirdal S, Ettema G (2009) Freely chosen pedal rate during free cycling on a roller and ergometer cycling. Eur J Appl Physiol 106:799-805

Leirdal S, Ettema G (2010) Pedalling technique and energy cost in cycling. Med Sci Sports Exerc. doi:10.1249/MSS.0b013e3181 f6b7ea (accepted 23 Aug 2010)

Lorås H, Leirdal S, Ettema G (2009) The muscle force component in pedalling retains constant direction across pedalling rates. J Appl Biomech 25:1-9

McGhie D, Ettema G (2011) The effect of cadence on timing of muscle activation and mechanical output in cycling: On the activation dynamics hypothesis. J Electromyogr Kinesiol 21:18-24

Medbø JI, Mamen A, Holth Olsen O, Evertsen F (2000) Examination of four different instruments for measuring blood lactate concentration. Scand J Clin Lab Invest 60:367-380
Millet GP, Tronche C, Fuster N, Candau R (2002) Level ground and inclined cycling efficiency in seated and standing positions. Med Sci Sport Exer 34:(10)1645-1652

Patterson RP, Pearson JL (1983) The influence of flywheel weight and pedalling frequency on the biomechanics and physiological response to bicycle exercise. Ergonomics 26(7):659-668

Price D, Donne B (1997) Effect of variation in seat tube angle at different seat heights on submaximal cycling performance in man. J Sport Sci 15:395-402

Pyne DB, Boston T, martin DT, Logan A (2000) Evaluation of the lactate Pro blood lactate analyser. Eur J Appl Physiol 82:112-116

Ricard MD, Hills-Meyer P, Miller MG, Michael TJ (2006) The effects of bicycle frame geometry on muscle activation and power during a Wingate anaerobic test. J. Sport Sci Med 5:25-32

Samozino P, Nicolas H, Hintzy F (2006) Interactions between cadence and power output effects on mechanical efficiency during sub maximal cycling exercises. Eur J Appl Physiol 97:133-139

Sanderson DJ (1991) The influence of cadence and power output on the biomechanics of force application during steady-rate cycling in competitive and recreational cyclists. J Sport Sci 9:191-203

Sanderson DJ, Black A (2003) The effect of prolonged cycling on pedal forces. J Sport Sci 21:191-199

Seabury JJ, Adams WC, Ramey MR (1977) Influence of pedalling rate and power output on energy expenditure during bicycle ergometry. Ergonomics 20(5):491-498

Umberger BR, Scheuchenzuber HJ, Manos TM (1998) Differences in power output during cycling at different seat tube angles. J Hum Move Stud 35:21-36

Welbergen E, Clijsen LPVM (1990) The influence of body position on maximal performance in cycling. Eur J Appl Physiol 61:138-142

Zameziati K, Mornieux DR, Belli A (2006) Relationship between the increase of effectiveness indexes and the increase of muscular efficiency with cycling power. Eur J Appl Physiol 96:274-281 